\title{
Breite Unterstützung für die Kampagne smarter medicine
}

Trägerschaft «smarter medicine»

Mitte Juni ist ein neuer Trägerverein gegründet worden, welcher der vor drei Jahren lancierten Kampagne smarter medicine in der Schweiz mehr Schub verleihen möchte. Nebst medizinischen Fach- und Berufsorganisationen unterstützen auch Verbände, welche die Interessen der Patienten und Konsumenten vertreten, die Stossrichtung der Kampagne. Sie möchten gemeinsam die Öffentlichkeit dafür sensibiliseren, dass bei gewissen Behandlungen manchmal weniger Medizin mehr Lebensqualität für die Betroffenen bedeuten kann.

Vor ein paar Jahren wurden in den USA Stimmen laut, welche vor den Auswirkungen der medizinischen Überversorgung («Overuse») warnten. 2011 lancierten Ärztinnen und Ärzte die «Choosing Wisely»-Initiative. Ziel dieser Initiative ist es, nicht nur «kluge Entscheidungen» herbeizuführen, sondern auch die offene Diskussion zwischen Ärzteschaft, den Patienten und der Öffentlichkeit zu fördern.

Kernstück von «Choosing Wisely» sind sogenannte «Top5-Listen» aus jeder klinischen Fachdisziplin. Diese Top5-Listen enthalten je fünf medizinische Massnahmen, die in der Regel unnötig sind. Das heisst: Ärzte und Patienten sollten miteinander darüber reden, ob nicht besser auf eine Behandlung verzichtet werden kann, weil die damit verbundenen Risiken potentiell grösser sind als der Nutzen.

Die Schweizerische Akademie der Medizinischen Wissenschaften (SAMW) hat die Choosing-Wisely-Initiative in ihrer Roadmap «Nachhaltiges Gesundheitssystem» propagiert. Bei der Schweizerischen Gesellschaft für Allgemeine Innere Medizin (SGAIM) stiess dieses Anliegen ebenfalls auf grosses Interesse. Eine Kommission machte sich in der Folge daran, eine eigene Top-5-Liste für den ambulanten Bereich in der Schweiz auszuarbeiten, und stellte diese 2014 unter dem Namen smarter medicine der Öffentlichkeit vor. Zwei Jahre später erfolgte die Publikation einer Top-5-Liste für den stationären Bereich. Trotzdem konnte die smarter medicine-Kampagne in der Schweiz - im Gegensatz zu anderen Ländern - noch nicht so recht Fuss fassen und wurde von anderen medizinischen Fachgesellschaften kaum mitgetragen. Deshalb ergriffen SGAIM und SAMW die Initiative, um eine grössere Trägerschaft für die Kampagne in der Schweiz zu bilden. Dabei sollten auch die Patient/innen und Konsu- ment/innen sowie die anderen Gesundheitsberufe eingebunden werden. Der Trägerverein smarter medicine wird nun im Juni 2017 gegründet. Neben SGAIM und SAMW sind auch der Schweizerische Verband der Berufsorganisationen im Gesundheitswesen (SVBG), der Dachverband der Schweizer Patientenstellen (DVSP) sowie die Konsumentenorganisationen Stiftung für Konsumentenschutz (SKS), Fédération Romande des Consommateurs (FRC) und Associazione consumatrici et consumatori della Svizzera italiana (acsi) als Mitglied dabei.

Der Trägerverein verfolgt folgende Ziele:

- Ausarbeitung und Publikation weiterer Top-5-Listen durch medizinische Fachgesellschaften etc. fördern;

- Die Verbindlichkeit der Empfehlungen erhöhen;

- Andere Gesundheitsberufe (interprofessioneller Ansatz) aktiv einbeziehen;

- Patient/innen und Kund/innen für das Anliegen sensibilisieren;

- Öffentliche Diskussion über Behandlungsqualität (Über- resp. Unterversorgung) anstossen;

- Thema Behandlungsqualität als Teil der medizinischen Weiter- und Fortbildung etablieren und verankern;

- Unterstützung der Kampagne durch Politik und Behörden erreichen.

Den Gründungsmitgliedern des Trägervereins ist es sehr wichtig, dass die Kampagne smarter medicine nicht von der Gesundheitsökonomie vereinnahmt wird. Es geht in erster Linie darum, die Behandlungsqualität nach dem Motto «Weniger Medizin kann mehr sein» zu verbessern.

Weitere Informationen zur Kampagne und zur Trägerschaft finden Sie unter www.smartermedicine.ch 